\title{
AN EXTENDED ANN-BASED HIGH SPEED ACCURATE TRANSMISSION LINE FAULT LOCATION FOR DOUBLE PHASE TO- EARTH FAULT ON NON-DIRECT-GROUND
}

\author{
Okwudili E. Obi ${ }^{1}$, Oseloka A. Ezechukwu' ${ }^{1}$, Chukwuedozie N. Ezema ${ }^{2}$ \\ ${ }^{1}$ Department of Electrical Engineering, Nnamdi Azikiwe University, Awka, Anambra State, \\ Nigeria \\ ${ }^{2}$ Department of Electronic and Computer Engineering, Nnamdi Azikiwe University, Awka, \\ Anambra State, Nigeria
}

DOI: https://doi.org/10.29121/IJOEST.v1.i1.2017.04

\begin{abstract}
This research has developed an extended Artificial Neural Networks (ANN) based high speed accurate transmission line fault location for double phase to- earth fault on non-direct-ground. Therefore, this research presents a system that capable of detecting and locating the fault with less proportion of error. This system uses the Global Positioning System (GPS) to locate the position and the Global System for Mobile Communication (GSM) to send these messages to system supervisor. A reduction in the size of the neural network improves the performance of the same and this can be achieved by performing feature extraction. By doing this, all of the important and relevant information present in the waveforms of the voltage and current signals can be used effectively. Voltage and current waveforms have been generated and were sampled at a frequency of 720 Hertz. The neural network diagnostic system trained for double faults was found to be able to accurately diagnose abnormal behavior resulting from simultaneous multiple faults. Graceful degradation of the diagnostic system was observed in situations where faults where not accurately diagnosed or under damage to a few nodes.
\end{abstract}

Keywords: Neural Network; Fault Diagnosis; Learning Strategies; Data Pre-Processing; Double Line - Ground Faults.

Cite This Article: Okwudili E. Obi, Oseloka A. Ezechukwu, \& Chukwuedozie N. Ezema. (2017). AN EXTENDED ANN-BASED HIGH SPEED ACCURATE TRANSMISSION LINE FAULT LOCATION FOR DOUBLE PHASE TO- EARTH FAULT ON NON-DIRECTGROUND. International Journal of Engineering Science Technologies, 1(1), 31-47. doi: 10.29121/IJOEST.v1.i1.2017.04

\section{INTRODUCTION}

A variety of methods of detecting and locating faults on power transmission lines exist. Most of these methods utilize the measurements from voltage and current transformers at substations or switching stations to perform their analyses. A review of common methods of fault location reveals that impedance-based and traveling wave-based fault location are the most common traditional methods.

The majority of systems rely upon three phase AC electric power is the product of two quantities: current and voltage (Cichoki \& Unbehauen, 2013). These two quantities can vary 
with respect to time (AC power) or can be kept at constant levels (DC power).Alternating current generators can produce a variable number of phases of power. A higher number of phases leads to more efficient power system operation but also increases the infrastructure requirements of the system. Power systems deliver energy to loads that perform a function. These loads range from household appliances to industrial machinery. Most loads expect a certain voltage and, for alternating current devices, a certain frequency and number of phases. The appliances found in homes, for example, will typically be single-phase operating at 50 or $60 \mathrm{~Hz}$ with a voltage between 11 and 260 volts (depending on national standards). All devices in your home will also have a wattage, this specifies the amount of power the device consumes. At any one time, the net amount of power consumed by the loads on a power system must equal the net amount of power produced by the supplies in addition to the power lost in transmission system (Anderson, 2015).

In these days high voltage transmission lines cover long distances, hundreds of kilometers, particularly when the line passes through hilly terrains. When a fault occurs on these transmission lines, it is extremely difficult to patrol the line from tower to tower to identify the faulty spot. Accurate location of faults does not only save the time but also saves different recourse for the power. Power system operator needs accurate information so that he can deploy men and machinery to the accurate spot immediately and rectify the fault thereby saving lot of time and resources. Software system, communication system such as SCADA and PLCC hardware system can be designed. For fault location data from SCADA such as oscillo graphs, relays and the sequence of events are used for fault location. Now available latest technology GPS can be used to locate a fault on long high voltage transmission lines. Self-monitoring hardware is configured at foundation sites for both conditions by inserting the information of a fault location (GPS) into Geographical information system computer (Das \& Novosel, 2013).

Therefore, this paper presents a system that capable of detecting and locating the fault with less proportion of error. This system uses the global positioning system (GPS) to locate the position and the global system for mobile (GSM) to send these messages to system supervisor.

\section{Neural Network Based Methods}

Neural networks have been put in use for fault diagnosis quite recently and have gained significant importance since Sobajic and Pao used neural networks for the prediction of critical clearing time (Eriksson and Rockefeller, 2015). Wide usage of neural networks started by late eighties and during early nineties. Neural networks are usually used to achieve greater efficiency in fault detection, classification and location. A lot of research has been done and abundant literatures have been published in the field of fault diagnosis using neural networks. Majority of the work made use of feed-forward multilayer perceptron technique. Kulicke and Dalstein used neural networks for the detection of faults on transmission lines and also differentiated between arcing and nonarcing faults. A new technique for the detection and location of high speed faults using neural networks has been proposed by Rikalo, Sobajic and Kezunovic (Cook, 2015). Neural network based single ended fault location techniques have been widely researched by Chen and Maun while Song used neural networks for fault diagnosis on series compensated lines. Other relevant work in the field of fault diagnosis using hybrid artificial neural-network modules can be found in these references (Kasztenny et al, 2011). 


\section{Artificial Neural Networks and Their Application in Transmission Line Fault Diagnosis}

An Artificial Neural Network (ANN) can be described as a set of elementary neurons that are usually connected in biologically inspired architectures and organized in several layers (Zhang et al, 2013). The structure of a feed-forward ANN, also known as the perceptron, is shown in Figure 1. There are Ni numbers of neurons in each ith layer and the inputs to these neurons are connected to the previous layer of neurons. The input layer is fed with the excitation signals. Simply put, an elementary neuron is like a processor that produces an output by performing a simple non-linear operation on its inputs (Girgis et al, 1992). A weight is attached to each and every neuron and training an ANN is the process of adjusting different weights tailored to the training set. An Artificial Neural Network learns to produce a response based on the inputs given by adjusting the node weights. Hence there is need for a set of data referred to as the training data set, which is used to train the neural network.

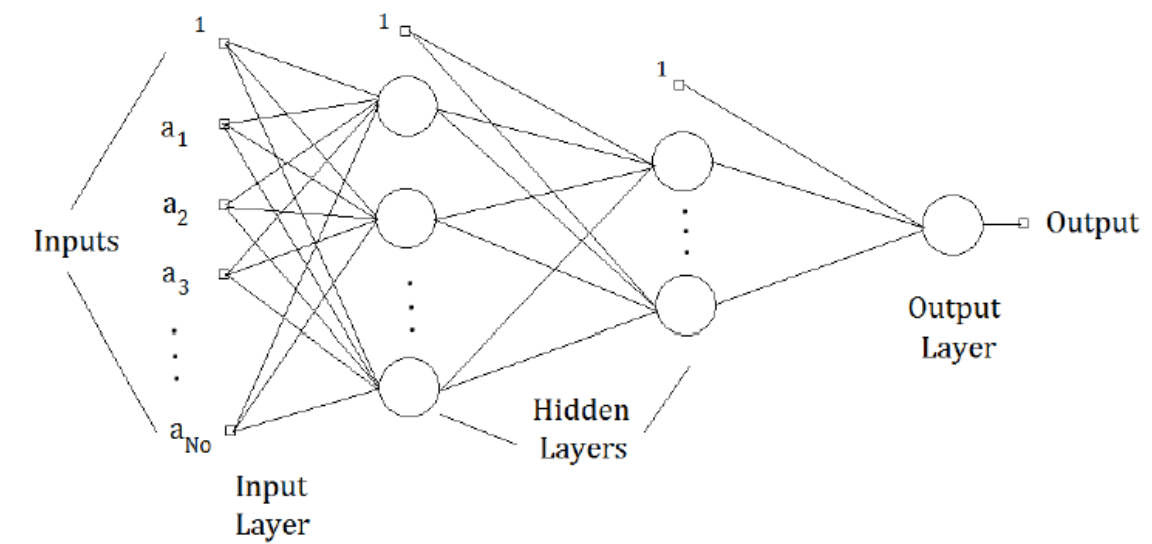

Figure 1: A basic three-layer architecture of a feed forward ANN. (Bouthiba, 2004)

In Figure $1, a_{1}, a_{2} \ldots a_{N 0}$ is the set of inputs to the ANN. Due to their outstanding pattern recognition abilities ANNs are used for several purposes in a wide variety of fields including signal processing, computers and decision making. Some important notes on hybrid artificial neural networks are (Saha et al, 2004):

- Either signal features extracted using certain measuring algorithms or even unprocessed samples of the input signals are fed into the ANN.

- The most recent along with a few older samples of the signals are fed into the ANN.

- The output provided by the neural network corresponds to the concerned decision which might be the type of fault, existence of a fault or the location of a fault.

- The most important factor that affects the functionality of the ANN is the training pattern that is employed.

- Pre-processing and post-processing techniques may be employed as well to enhance the learning process and reduce the training time of the ANN.

One of the biggest drawbacks of applications that make use of artificial neural networks is that no well-defined guide exists to help in choosing the ideal number of hidden layers to be used and the number of neurons per each hidden layer. From a different perspective, it is advantageous considering the ability to generalize (Wanjing \& Yuan, 2012). A vital feature of ANN is its dedication to parallel computing. Hence it can produce a correct output corresponding to any input even if the concerned input was not fed into the ANN during the training process. Another challenge in the ANN based application development was to synthesize the algorithm for the 
adaptive learning process. The back error-propagation algorithm is the basic algorithm in which the neuron weights are adjusted in consecutive steps to minimize the error between the actual and the desired outputs. This process is known as supervised learning.

\section{Learning Strategies}

The basic concept behind the successful application of neural networks in any field is to determine the weights to achieve the desired target and this process is called learning or training. The two different learning mechanisms usually employed are supervised and unsupervised learning. In the case of supervised learning, the network weights are modified with the prime objective of minimization of the error between a given set of inputs and their corresponding target values (Karl \& David, 2015). Hence the training data set is known, which is a set of inputs and the corresponding targets, the neural network should output ideally. This is called supervised learning because both the inputs and the expected target values are known prior to the training of ANN.

On the other hand, in the case of unsupervised learning, we are unaware of the relationship between the inputs and the target values are not known. The neural network is trained with a training data set in which only the input values are known. Hence it is very important to choose the right set of examples for efficient training. These examples are usually chosen using some sort of a similarity principle (Takagi et al, 1982). The most commonly used unsupervised learning algorithms are the Self-Organizing Map (SOM) and the Adaptive Resonance Theory (ART). The learning strategy employed depends on the structure of the neural network. Feed forward networks are trained using the supervised learning strategy.

\section{METHODOLOGY}

\section{Data Pre-Processing}

A reduction in the size of the neural network improves the performance of the same and this can be achieved by performing feature extraction. By doing this, all of the important and relevant information present in the waveforms of the voltage and current signals can be used effectively. Voltage and current waveforms have been generated and were sampled at a frequency of 720 Hertz. The voltage and current samples of all the three phases are noted along with the corresponding pre-fault values.

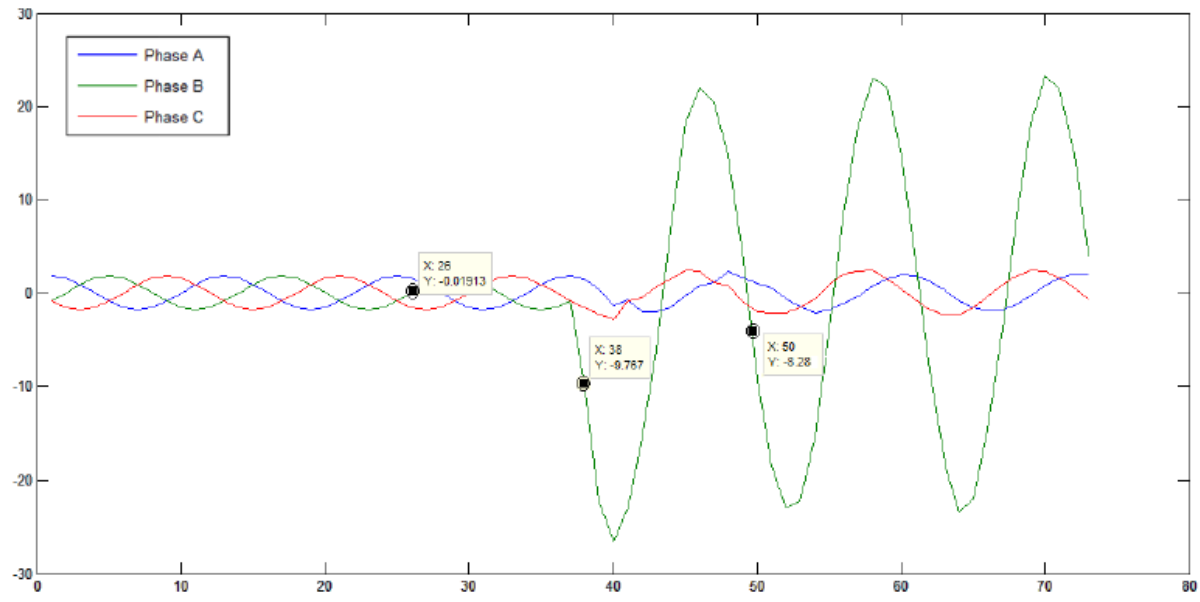

Figure 2: Data pre-processing illustration. 
Figure 2 shows the current waveform of a Phase B - ground fault at a distance of $60 \mathrm{~km}$ from terminal A on a $300 \mathrm{~km}$ transmission line. The waveform is the plot of the samples sampled at a frequency of $720 \mathrm{~Hz}$. Hence there are 12 samples per each cycle.

Now, the 50th sample (12th sample after the occurrence of the fault) on phase B is noted along with the 26th sample (12th sample before the occurrence of the fault, corresponding to the postfault sample considered). Once this is done, the inputs to the neural network are the ratios of the voltages and currents in each of the phases before and after the occurrence of fault as shown in Table 1. The inputs in matrix format are shown below:

$$
\left[\begin{array}{c}
V_{a}(n+12) / V_{a}(n-12) \\
V_{b}(n+12) / V_{b}(n-12) \\
V_{c}(n+12) / V_{c}(n-12) \\
I_{a}(n+12) / I_{a}(n-12) \\
I_{b}(n+12) / I_{b}(n-12) \\
I_{c}(n+12) / I_{c}(n-12)
\end{array}\right]
$$

Where ' $\mathrm{n}=38$ ' is the sample at which fault occurs.

Hence, there is a set of six inputs each time ( 3 for the phase voltages and 3 for the phase currents) to all the neural networks discussed in this work. Care has been taken each time to make sure the denominator of each of the inputs is non-zero. If it is zero, the value of $n$ is incremented by 1 and the next sample is taken into consideration for the entire process. The advantage of performing this scaling is to reduce the training computation time. For the sake of illustration, the Table 1 shows the voltage and current values that are scaled with respect to their pre-fault values and used as a part of the training set. In Table 1, Va, Vb and Vc are the post fault voltage and current sample values and $\mathrm{Va}(\mathrm{pf}), \mathrm{Vb}(\mathrm{pf})$ and $\mathrm{Vc}(\mathrm{pf})$ are the corresponding pre-fault values as illustrated earlier. The given table depicts the values for all the various types of faults and also during the no fault case. The fault has been simulated on a $300 \mathrm{~km}$ long transmission line at a distance of $100 \mathrm{~km}$ from the terminal A.

\section{Training Process}

Two important steps in the application of neural networks for any purpose are training and testing. The first of the two steps namely training the neural network is discussed in this section. Training is the process by which the neural network learns from the inputs and updates its weights accordingly. In order to train the neural network we need a set of data called the training data set which is a set of input output pairs fed into the neural network. Thereby, we teach the neural network what the output should be, when that particular input is fed into it. The ANN slowly learns the training set and slowly develops an ability to generalize upon this data and will eventually be able to produce an output when a new data is provided to it. During the training process, the neural network's weights are updated with the prime goal of minimizing the performance function. This performance function can be user defined, but usually feed forward networks employ Mean Square Error as the performance function and the same is adopted throughout this work.

As already mentioned in the previous chapter, all the voltages and currents fed into the neural network are scaled with respect to the corresponding voltage and current values before the 
occurrence of the fault. The outputs, depending upon the purpose of the neural network might be the fault condition, the type of fault or the location of the fault on the transmission line.

For the task of training the neural networks for different stages, sequential feeding of input and output pair has been adopted. In order to obtain a large training set for efficient performance, each of the ten kinds of faults has been simulated at different locations along the considered transmission line. In view of all these issues, about 100 different fault cases for each of the 10 kinds of faults have been simulated.

Apart from the type of fault, the phases that are faulted and the distance of the fault along the transmission line, the fault resistance also has been varied to include several possible real-time fault scenarios.

- The fault resistance has been varied as follows: $0.25 \mathrm{ohm}, 0.5 \mathrm{ohm}, 0.75 \mathrm{ohm}, 1 \mathrm{ohm}, 5$ ohm, 10 ohm, 25 ohm, 50 ohm.

- Fault distance has been varied at an incremental factor of every $3 \mathrm{~km}$ on a $300 \mathrm{~km}$ transmission line.

Table 1: Sample of Inputs to the neural network for various fault cases.

\begin{tabular}{|c|c|c|c|c|c|c|c|}
\hline \multirow{2}{*}{$\begin{array}{l}\text { Case } \\
\text { No: }\end{array}$} & \multicolumn{6}{|c|}{ Input Vector } & \multirow{2}{*}{$\begin{array}{l}\text { Fault } \\
\text { Type }\end{array}$} \\
\hline & $\mathrm{V}_{\mathrm{a}} / \mathrm{V}_{\mathrm{a}}(\mathrm{pf})$ & $\mathrm{V}_{\mathrm{b}} / \mathrm{V}_{\mathrm{b}}(\mathrm{pf})$ & $\mathrm{V}_{\mathrm{c}} / \mathrm{V}_{\mathrm{c}}(\mathrm{pf})$ & $\mathrm{I}_{\mathrm{a}} / \mathrm{I}_{\mathrm{a}}(\mathrm{pf})$ & $\mathrm{I}_{\mathrm{b}} / \mathrm{I}_{\mathrm{b}}(\mathrm{pf})$ & $\mathrm{I}_{\mathrm{c}} / \mathrm{I}_{\mathrm{c}}(\mathrm{pf})$ & \\
\hline 1 & 0.6204 & 0.9719 & 1.0425 & 1.6840 & 0.5056 & 0.8775 & $\begin{array}{l}\text { A to } \\
\text { Ground }\end{array}$ \\
\hline 2 & 0.6573 & 0.7351 & 0.8289 & 0.4024 & 27.6875 & 1.7453 & $\begin{array}{l}\text { B to } \\
\text { Ground }\end{array}$ \\
\hline 3 & 1.2580 & 0.9141 & 0.7924 & 1.4994 & -1.5179 & -4.7497 & $\begin{array}{l}\mathrm{C} \text { to } \\
\text { Ground }\end{array}$ \\
\hline 4 & -0.1882 & 0.6041 & 1.0001 & 4.9014 & 20.6762 & 0.9994 & A to $B$ \\
\hline 5 & 1.0000 & 0.5516 & 0.3276 & 1.0000 & 33.8158 & -7.1187 & B to $\mathrm{C}$ \\
\hline 6 & 1.1586 & 1.000 & 0.9208 & -1.6037 & 1.0025 & -2.2493 & $\mathrm{C}$ to $\mathrm{A}$ \\
\hline 7 & -0.1276 & 0.5841 & 0.9042 & 2.9694 & 30.4194 & 1.4733 & $\begin{array}{l}\text { A to B to } \\
\text { Ground }\end{array}$ \\
\hline 8 & 0.9359 & 0.5145 & 0.3833 & 0.9257 & 35.3006 & -6.7506 & $\begin{array}{l}\mathrm{B} \text { to } \mathrm{C} \text { to } \\
\text { Ground }\end{array}$ \\
\hline 9 & 0.9864 & 0.9147 & 0.8350 & 0.6229 & -1.2876 & -5.0284 & $\begin{array}{l}\mathrm{C} \text { to } \mathrm{A} \text { to } \\
\text { Ground }\end{array}$ \\
\hline 10 & 0.3135 & 0.4373 & 0.4991 & 1.8649 & 35.9958 & -6.5793 & $\begin{array}{c}\text { A to } B \text { to } \\
\text { C }\end{array}$ \\
\hline 11 & 1.0000 & 1.0001 & 1.0001 & 1.0000 & 1.0007 & 0.9998 & No Fault \\
\hline
\end{tabular}




\section{Testing Process}

As already mentioned in the previous section, the next important step to be performed before the application of neural networks is to test the trained neural network. Testing the artificial neural network is very important in order to make sure the trained network can generalize well and produce desired outputs when new data is presented to it.

There are several techniques used to test the performance of a trained network, a few of which are discussed in this section. One such technique is to plot the best linear regression fit between the actual neural network's outputs and the desired targets. Analyzing the slope of this line gives us an idea on the training process. Ideally the slope should be 1. Also, the correlation coefficient (r), of the outputs and the targets measures how well the ANN's outputs track the desired targets. The closer the value of ' $r$ ' is, to 1 , the better the performance of the neural network. Another technique employed to test the neural network is to plot the confusion matrix and look at the actual number of cases that have been classified positively by the neural network. Ideally this percentage is a 100 which means there has been no confusion in the classification process. Hence if the confusion matrix indicates very low positive classification rates, it indicates that the neural network might not perform well. The last and a very obvious means of testing the neural network is to present it with a whole new set of data with known inputs and targets and calculate the percentage error in the neural networks output. If the average percentage error in the ANN's output is acceptable, the neural network has passed the test and can be readily applied for future use.

The Neural Network toolbox in Simulink by the "MathWorks" divides the entire set of data provided to it into three different sets namely the training set, validation set and the testing set. The training data set as indicated above is used to train the network by computing the gradient and updating the network weights. The validation set is provided during to the network during the training process (just the inputs without the outputs) and the error in validation data set is monitored throughout the training process. When the network starts over fitting the data, the validation errors increase and when the number of validation fails increase beyond a particular value, the training process stops to avoid further over fitting the data and the network is returned at the minimum number of validation errors. The test set is not used during the training process but is used to test the performance of the trained network. If the test set reaches the minimum value of MSE at a significantly different iteration than the validation set, then the neural network will not be able to provide satisfactory performance.

\section{DISCUSSION OF RESULTS}

\section{Double Line - Ground Faults}

The design, development and performance of neural networks for the purpose of Double Line Ground fault location are discussed in this section. The third category of faults is the double line - ground faults. Three possible double line - ground faults exist which are denoted as ABG, BCG and ACG (based on which two of the three phases A,B and C are faulted).

\section{Training the Neural Network For Double Line - Ground Fault Location}

Feed forward back - propagation algorithm was once again used for the purpose of double line ground fault location on transmission lines. The reason for doing so, as already mentioned is that 
these networks perform very efficiently when there is availability of a sufficiently large training data set. For the purpose of training the neural network, several double line - ground faults have been simulated on the modeled transmission line on each of the three phases. The various factors that were varied were the fault distance (incremented by $3 \mathrm{~km}$ each time), the fault resistance (one of the chosen eight different fault resistances) and the phases that were faulted. About 100 fault cases were simulated for each phase with each of the eight different resistances as $0.25,0.5$, $0.75,1,5,10,25$ and $50 \mathrm{ohms}$ respectively. Hence a total of 2400 fault cases were simulated on the transmission line. In each of these cases, the voltage and current samples on all three phases (scaled with respect to their pre-fault values) are fed as inputs to the neural network. The neural network's output is the distance to the fault from terminal A. Thus each input output pair fed into the neural network has a set of six inputs and one output.

An exhaustive survey on various neural networks has been performed by varying the number of hidden layers and the number of neurons per hidden layer. A few neural networks that achieved satisfactory performance are presented first along with their error performance plots. Of these ANNs, the most appropriate ANN is chosen based on its Mean Square Error performance and the Regression coefficient of the Outputs vs. Targets.

Figures $4.42-4.45$ show the MSE and the Test phase performance plots of the neural networks $6-10-1$ and $6-20-1$ with 1 hidden layer. Figures $4.46-4.49$ show the MSE and the Test phase performance plots of the neural network $6-10-5-1$ and $6-21-11-1$ with 2 hidden layers.

Figure 3a shows the performance of the neural network (in terms of training, testing and validation) with 6 neurons in the input layer, 10 neurons in the hidden layer and 1 neuron in the output layer $(6-10-1)$. It can be seen that the best MSE performance of this neural network is 0.0047967 which is below the MSE goal of 0.01 (denoted by the black dotted line). It was found that the correlation coefficient between the outputs and the targets was 0.98193 in this case.

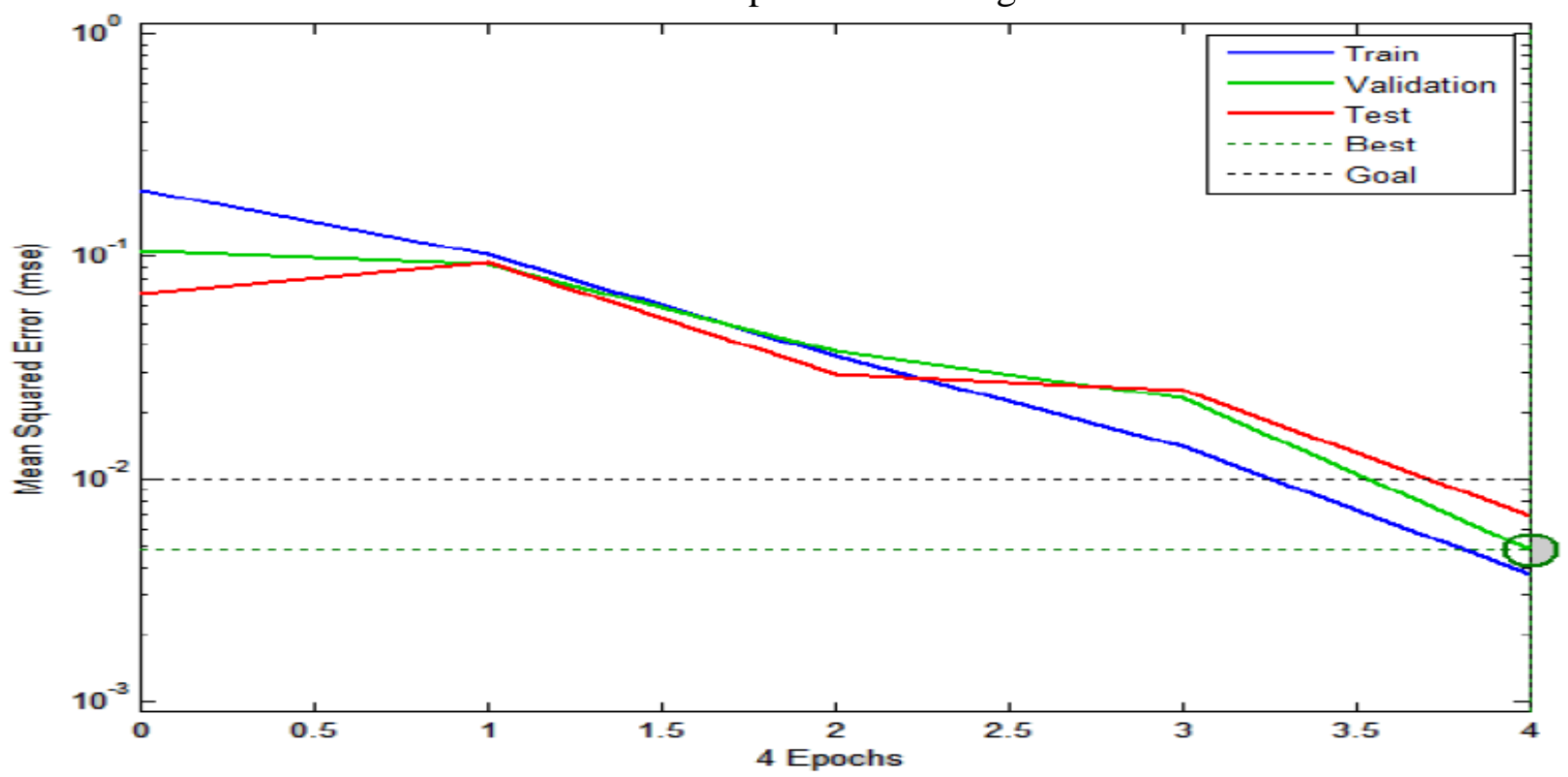

Figure 3a: Mean Square Error performance of the ANN with configuration (6-10-1). 
In order to test the performance of this network, 12 different double line - ground faults have been simulated on different phases with the fault distance being incremented by $25 \mathrm{Km}$ in each case and the percentage error in ANN's output has been calculated. Figure $3 \mathrm{~b}$ shows the results of this test conducted on the neural network (6-10-1). It can be seen that the maximum error is higher than 5 percent which is exorbitantly high.

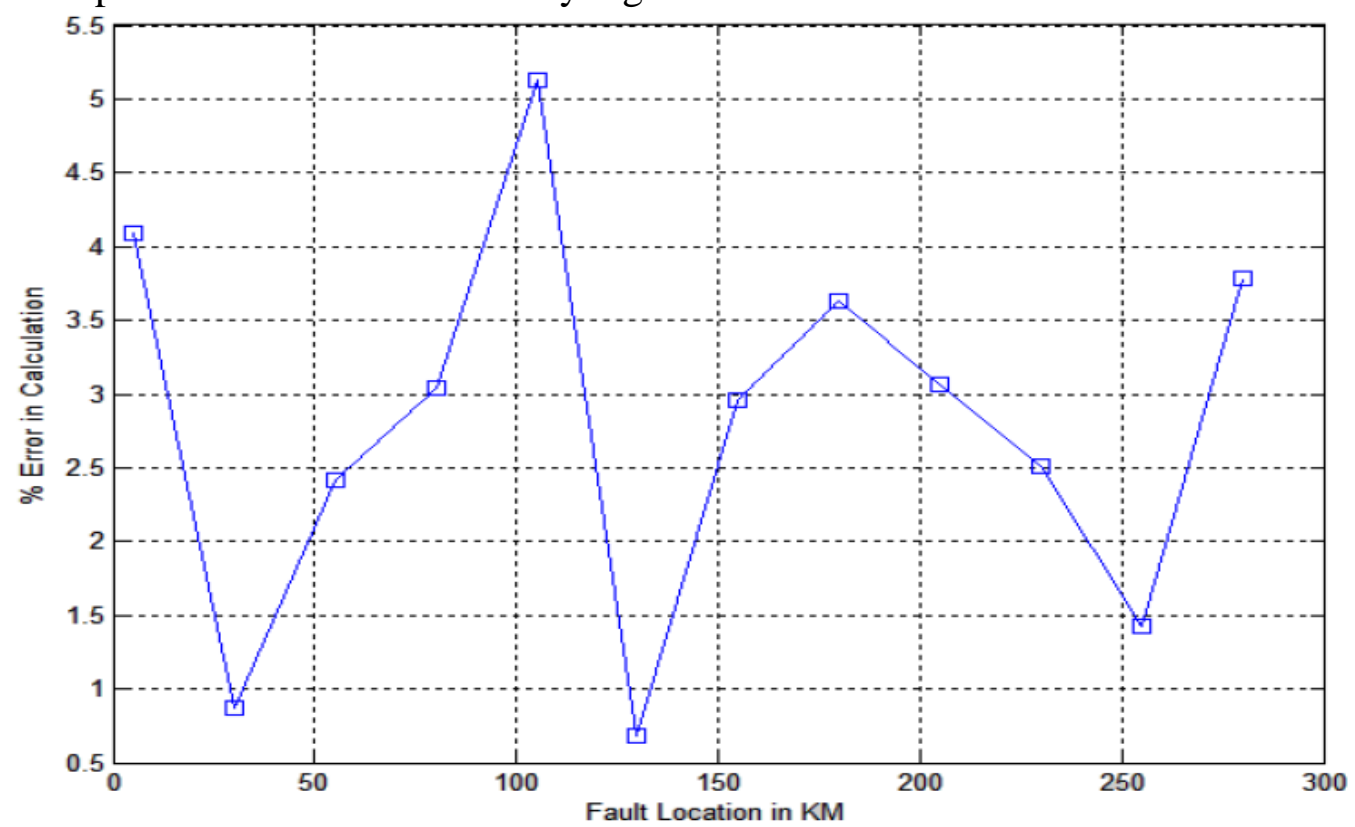

Figure 3b: Test Phase performance of the ANN with configuration (6-10-1).

Figure 4a shows the performance of the neural network (in terms of training, testing and validation) with 6 neurons in the input layer, 20 neurons in the hidden layer and 1 neuron in the output layer $(6-20-1)$. It can be seen that the best MSE performance of this neural network is 0.0013561 which is below the MSE goal of 0.01 (denoted by the black dotted line in the figure). It was found that the correlation coefficient between the outputs and the targets was 0.98804 for this neural network.

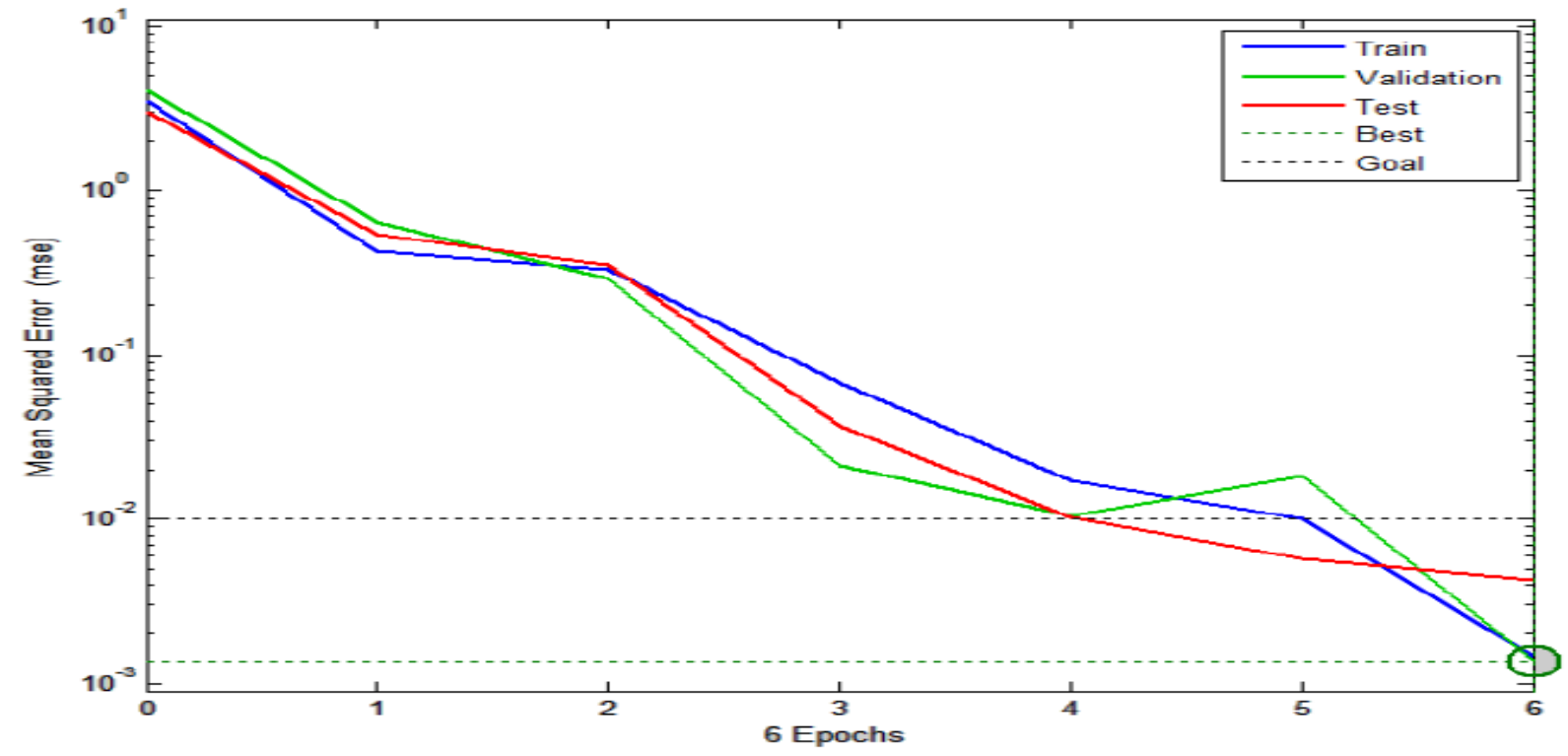

Figure 4a: Mean Square Error performance of the ANN with configuration (6-20-1). 


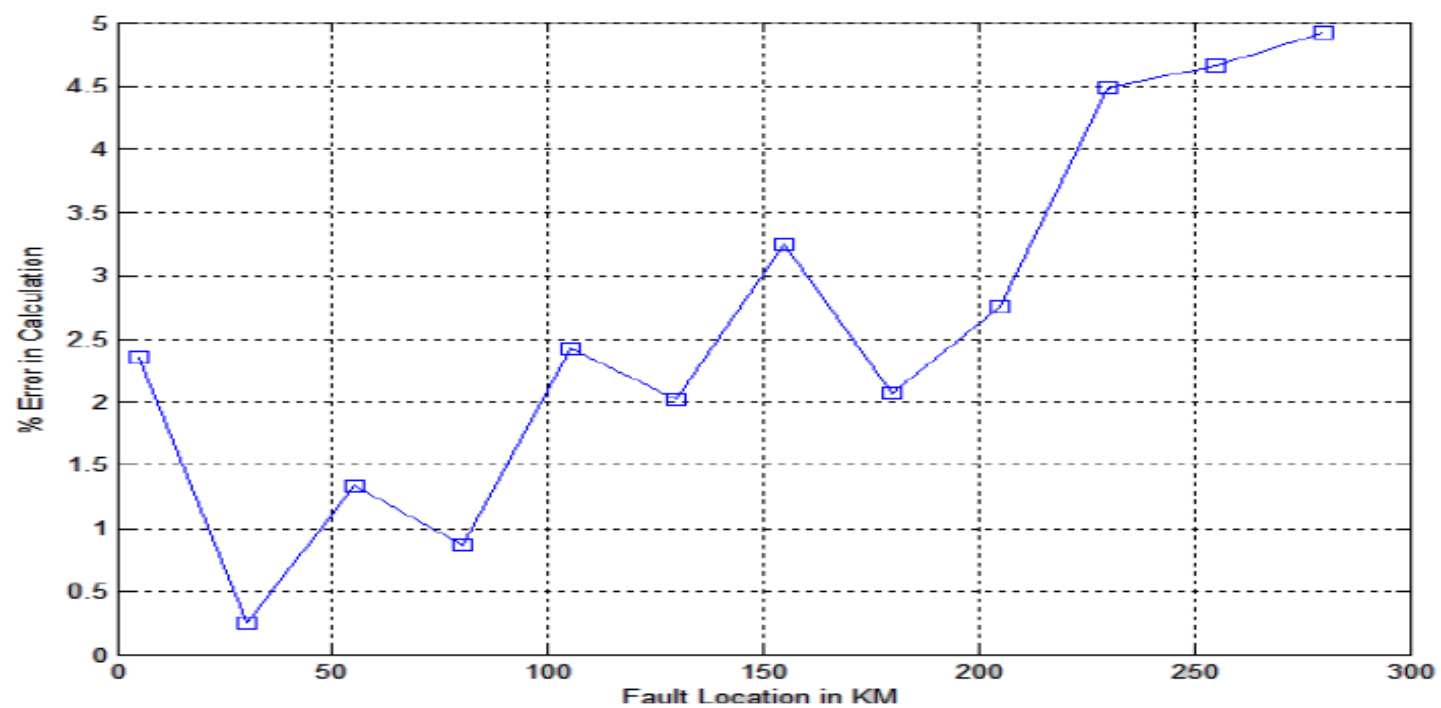

Figure 4b: Test Phase performance of the ANN with configuration (6-20-1).

In order to test the performance of this network the same method adopted for the earlier case is followed. 12 different double line - ground faults have been simulated on different phases with the fault distance being incremented by $25 \mathrm{Km}$ in each case and the percentage error in ANN's output has been calculated. Figure $4 \mathrm{~b}$ shows the results of this test conducted on the neural network (6-20-1). It is to be noted that the maximum error is higher than 4.75 percent which is too high for this purpose.

Figure 5 shows the performance of the neural network (in terms of training, testing and validation) with 6 neurons in the input layer, 2 hidden layers with 10 and 5 neurons in them respectively and 1 neuron in the output layer $(6-10-5-1)$. It can be seen that the best MSE performance of this neural network is 0.00338785 which is below the MSE goal of 0.01 (denoted by the black dotted line in the figure). It was found that the correlation coefficient between the outputs and the targets was 0.98913 for this neural network.

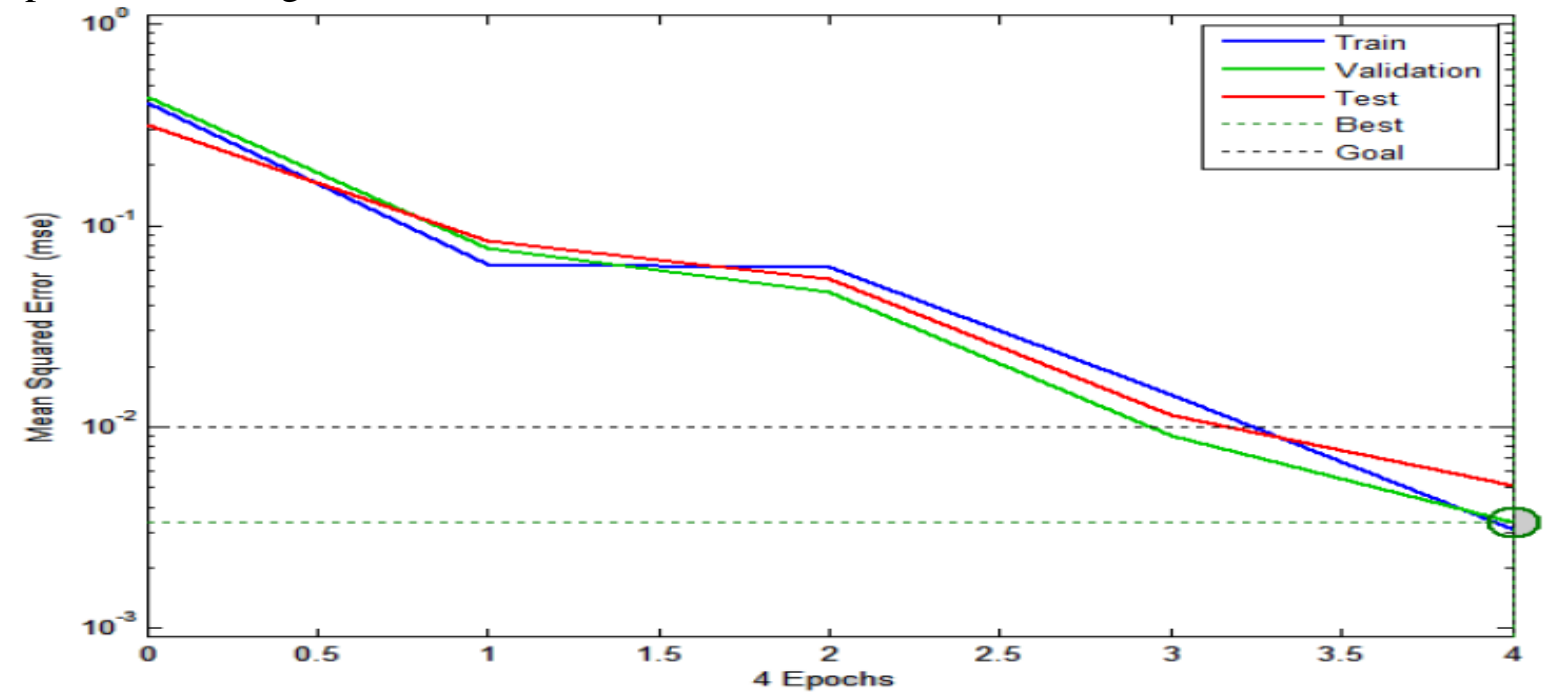

Figure 5: Mean Square Error performance of the neural network with configuration (6-10-5-1). 
In order to test the performance of this network the same method adopted for the earlier case is followed. 12 different double line - ground faults have been simulated on different phases with the fault distance being incremented by $25 \mathrm{Km}$ in each case and the percentage error in ANN's output has been calculated. Figure 6 shows the results of this test conducted on the neural network (6-10-5-1). It is to be noted that the maximum error is higher than 3.5 percent which is still not satisfactory for this purpose.

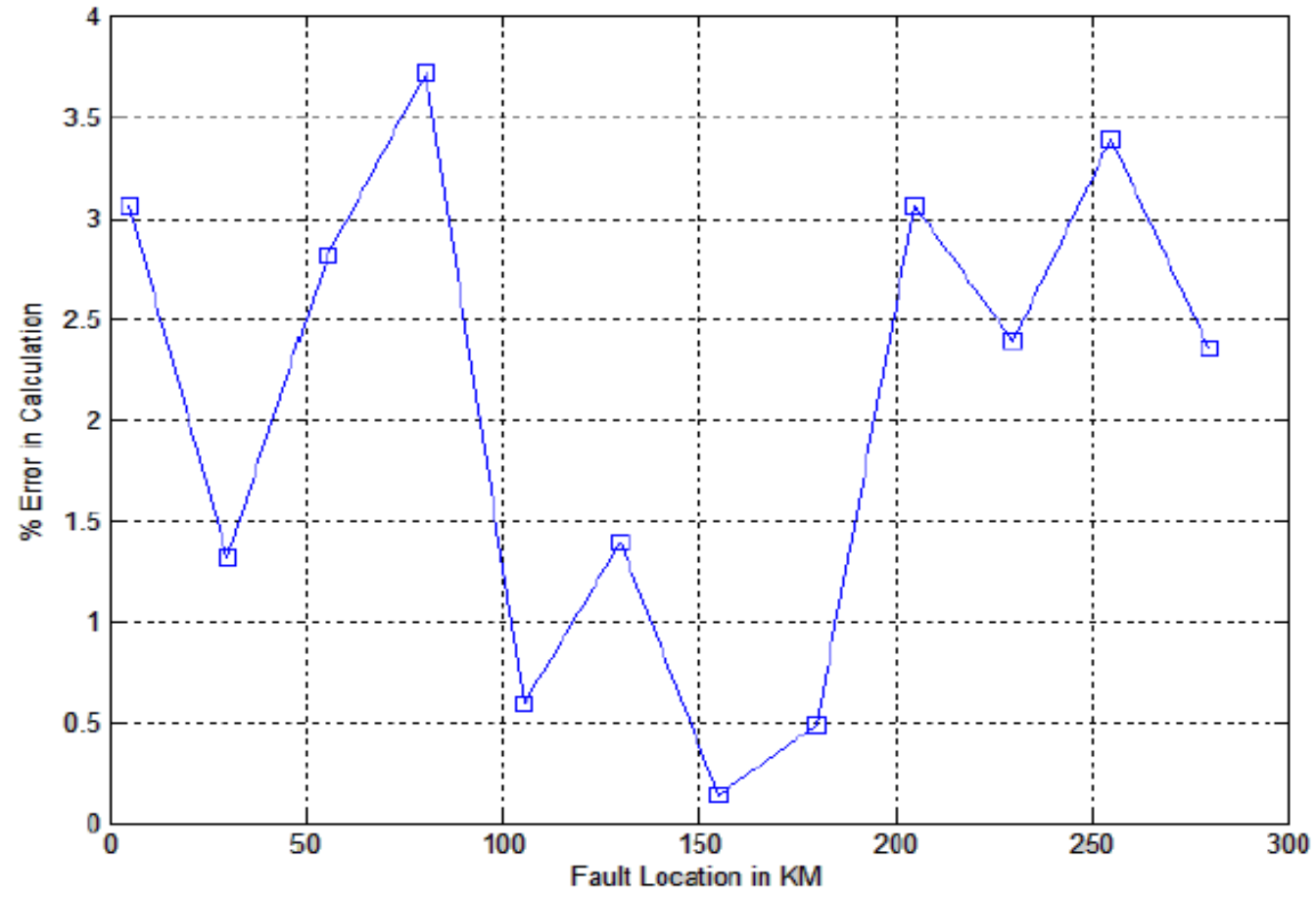

Figure 6: Test Phase performance of the ANN (6-10-5-1).

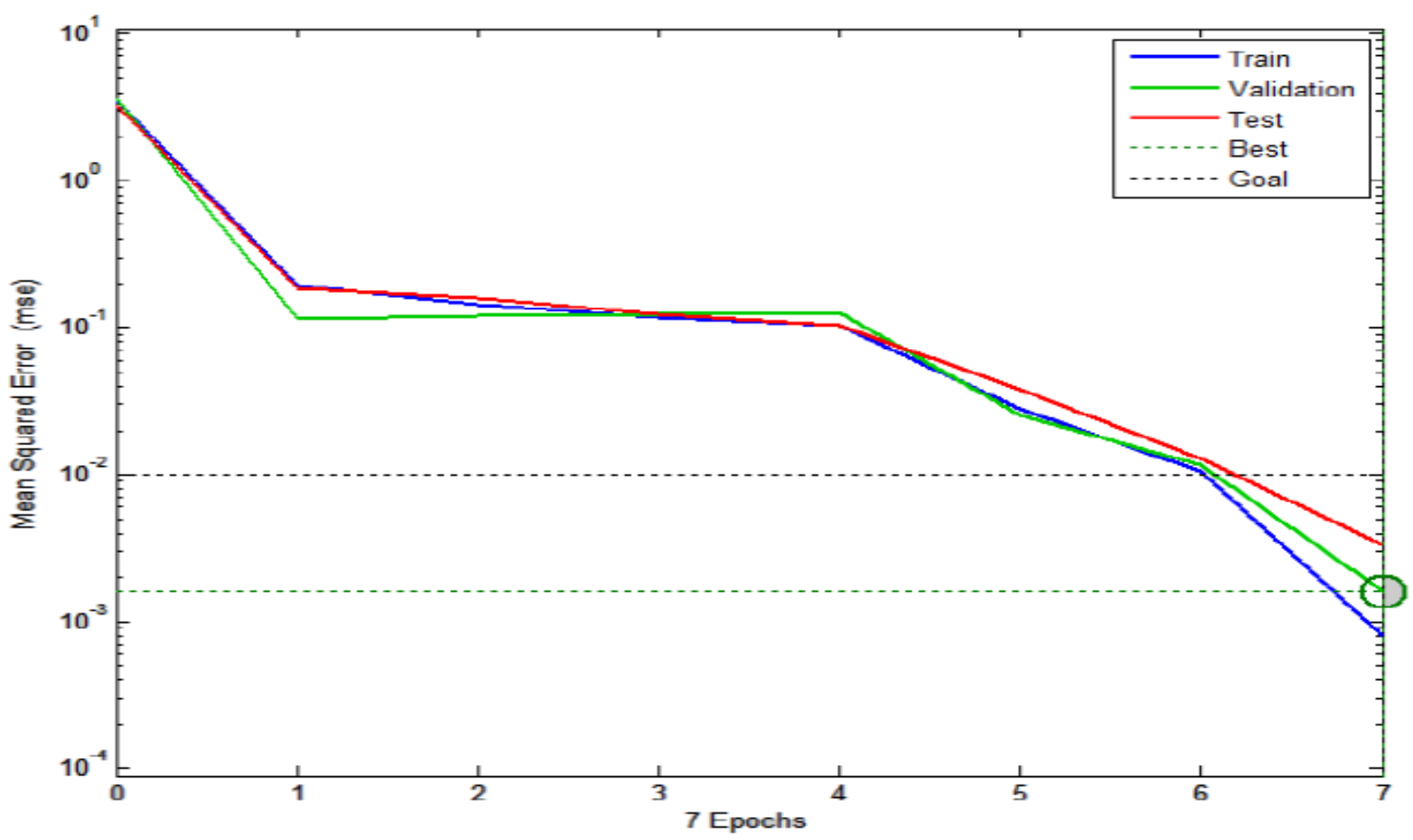

Figure 7: Mean Square Error performance of the neural network with configuration (6-21-11-1). 
Figure 7 shows the performance of the neural network (in terms of training, testing and validation) with 6 neurons in the input layer, 2 hidden layers with 21 and 11 neurons in them respectively and 1 neuron in the output layer $(6-21-11-1)$. It can be seen that the best MSE performance of this neural network is 0.00159395 which is below the MSE goal of 0.01 (denoted by the black dotted line in the figure). It was found that the correlation coefficient between the outputs and the targets was 0.99329 for this neural network which indicates very good regression fit.

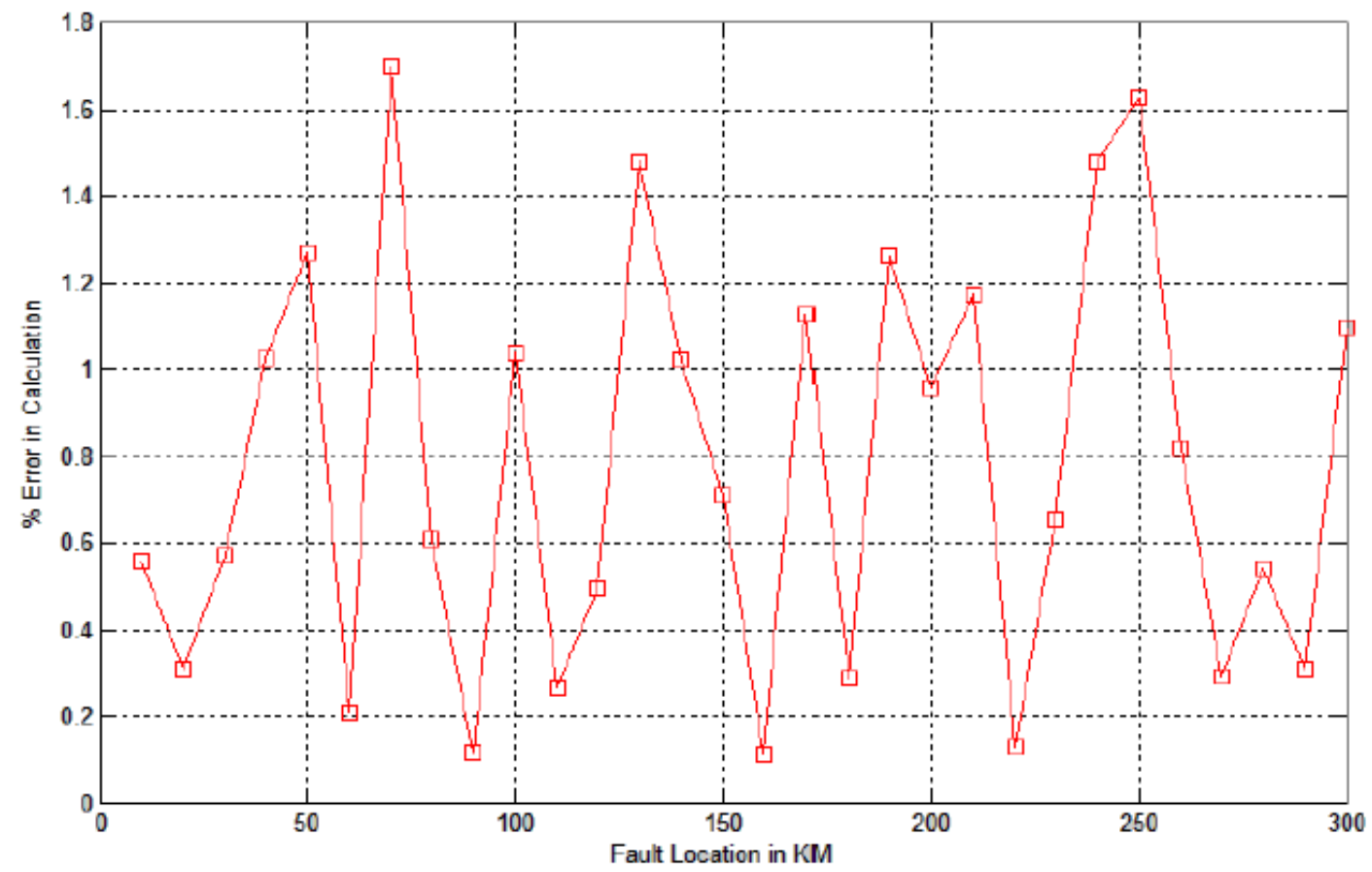

Figure 8: Test phase performance of the ANN (6-21-11-1).

In order to test the performance of this network, 100 different double line - ground faults have been simulated on different phases with the fault distance being incremented by $10 \mathrm{Km}$ in each case and the percentage error in calculated output has been calculated. Figure 8 shows the results of this test conducted on the neural network (6-21-11-1). It can be seen that the maximum error is around 1.71 percent which is very satisfactory. It is to be noted that the average error in fault location is just 0.863 percent. Hence, this neural network has been chosen as the ideal network for the purpose of double line - ground fault location on transmission lines.

Figure 9 shows an overview of the chosen ANN and it can be seen that the training algorithm used is Levenberg - Marquardt algorithm. The performance function chosen for the training process is mean square error. Figure 10 plots the best linear regression fit between the outputs and the targets. As already mentioned, the correlation coefficient in this case is found to be 0.99329 which is very good. 

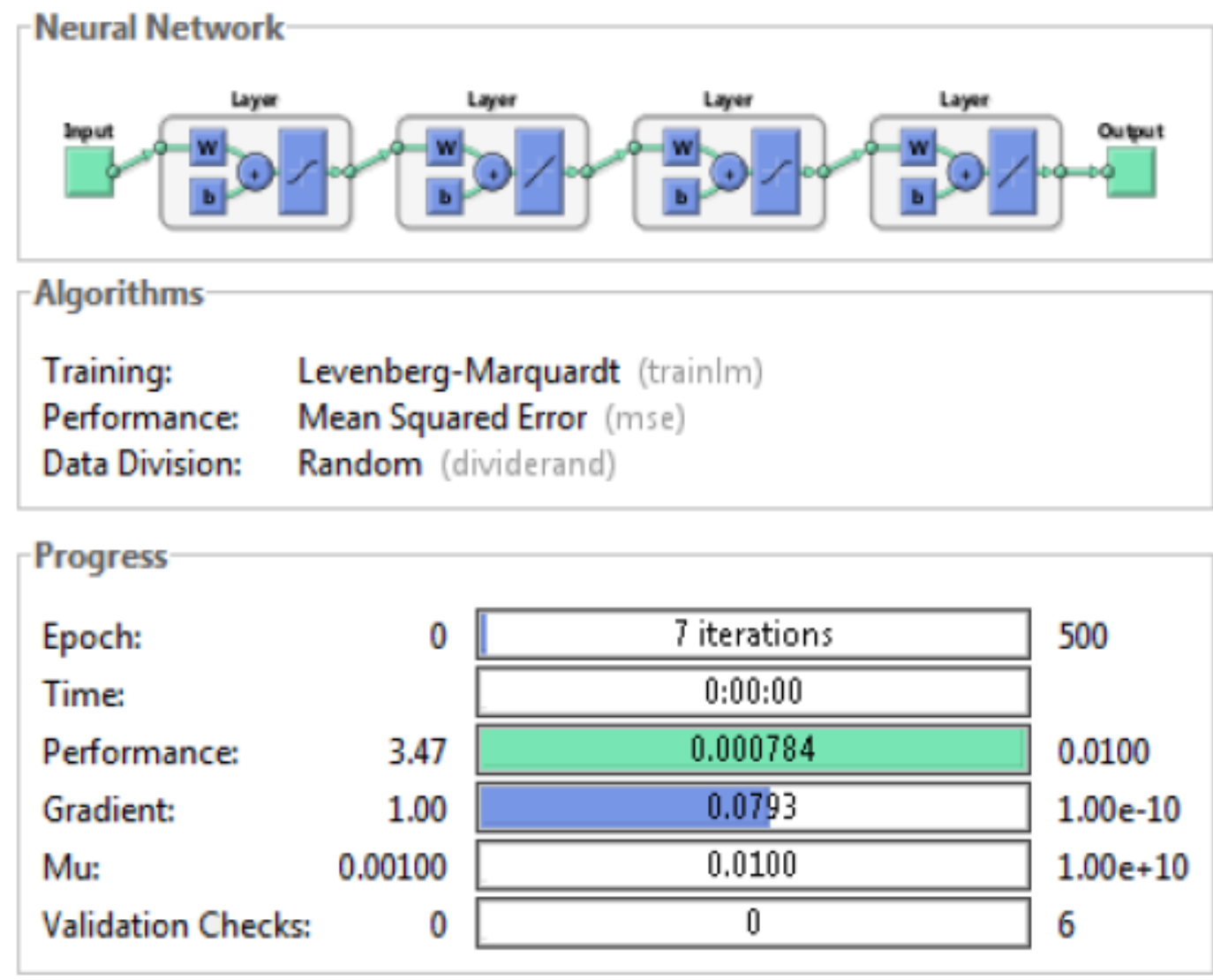

Figure 9: Overview of the chosen ANN (6-21-11-1) for Double Line-Ground Faults.

\section{Testing the Neural Network for Double Line - Ground Fault Location}

Now that the neural network has been trained, the next important step is to analyze the performance of this network which is called testing. The methods and means by which this neural network has been tested are discussed here under. One important factor that helps test the network is the test phase performance plot as shown in Figure 10.

It is to be noted that both the average as well as the maximum error percentages are in acceptable levels and hence the networks performance is satisfactory. Another means of determining the efficiency of a trained neural network is to check the gradient and validation performance plot as shown in Figure 13. It can be seen that there is a steady decrease in the gradient and also that the maximum number of validation fails is 3 during the training process. This indicates efficient training because the validation phase follows the test phase closely if the number of validation fails is low. This further implies that the neural network can generalize new data fed into it more effectively. 


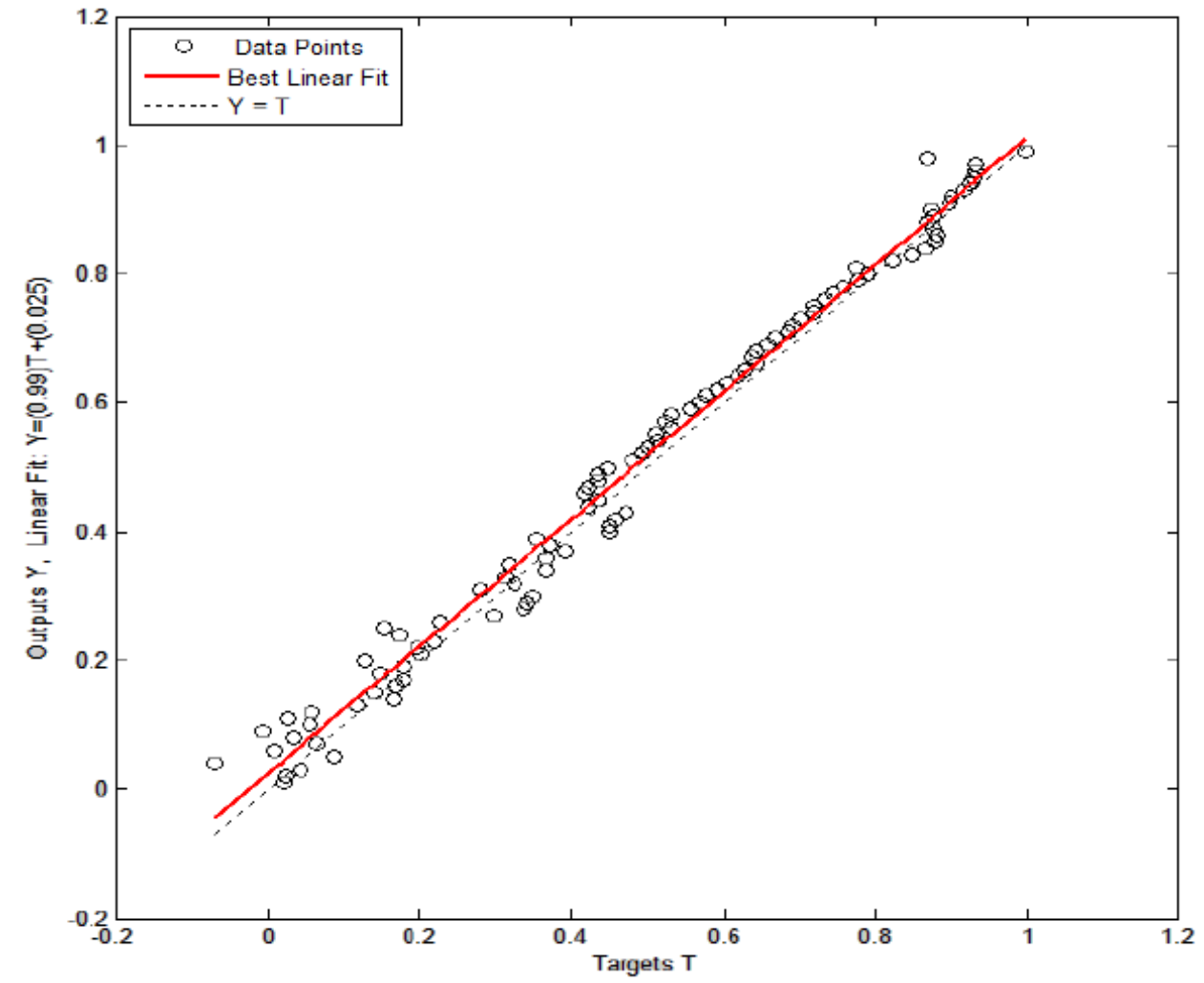

Figure 10: Regression fit of the outputs versus targets with configuration (6-21-11-1).
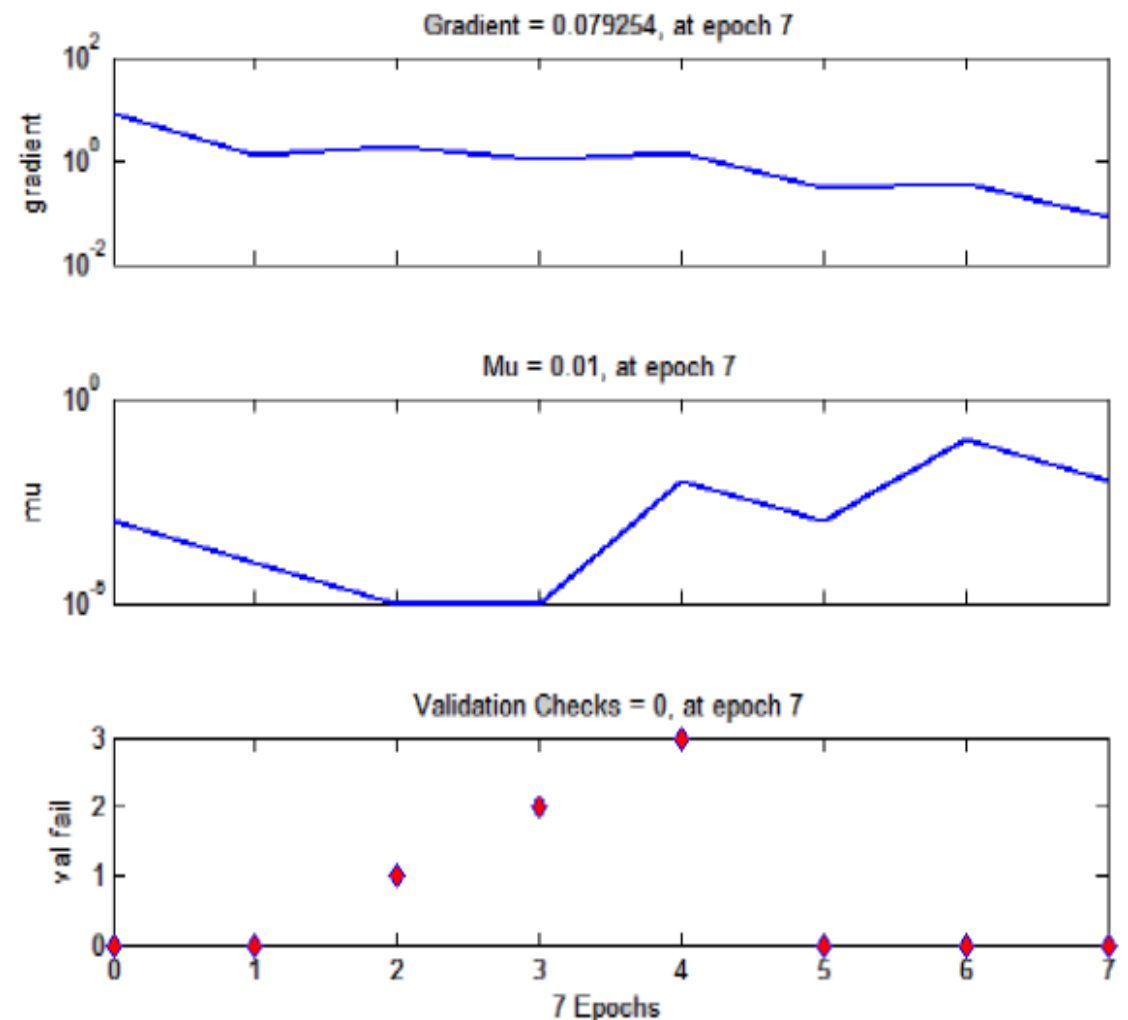

Figure 11: Gradient and validation performance plot of ANN with configuration (6-21-11-1). 
The third factor that is considered while evaluating the performance of the network is the correlation coefficient of each of the various phases of training, validation and testing. Figure 12 shows the regression plots of the various phases such as training, testing and validation. It can be seen that the best linear fit very closely matches the ideal case with an overall correlation coefficient of 0.99329 .

Figure 13 shows the structure of the chosen ANN for double line - ground fault location with 6 neurons in the input layer, 2 hidden layers with 21 and 11 neurons in them respectively and 1 neuron in the output layer $(6-21-11-1)$.
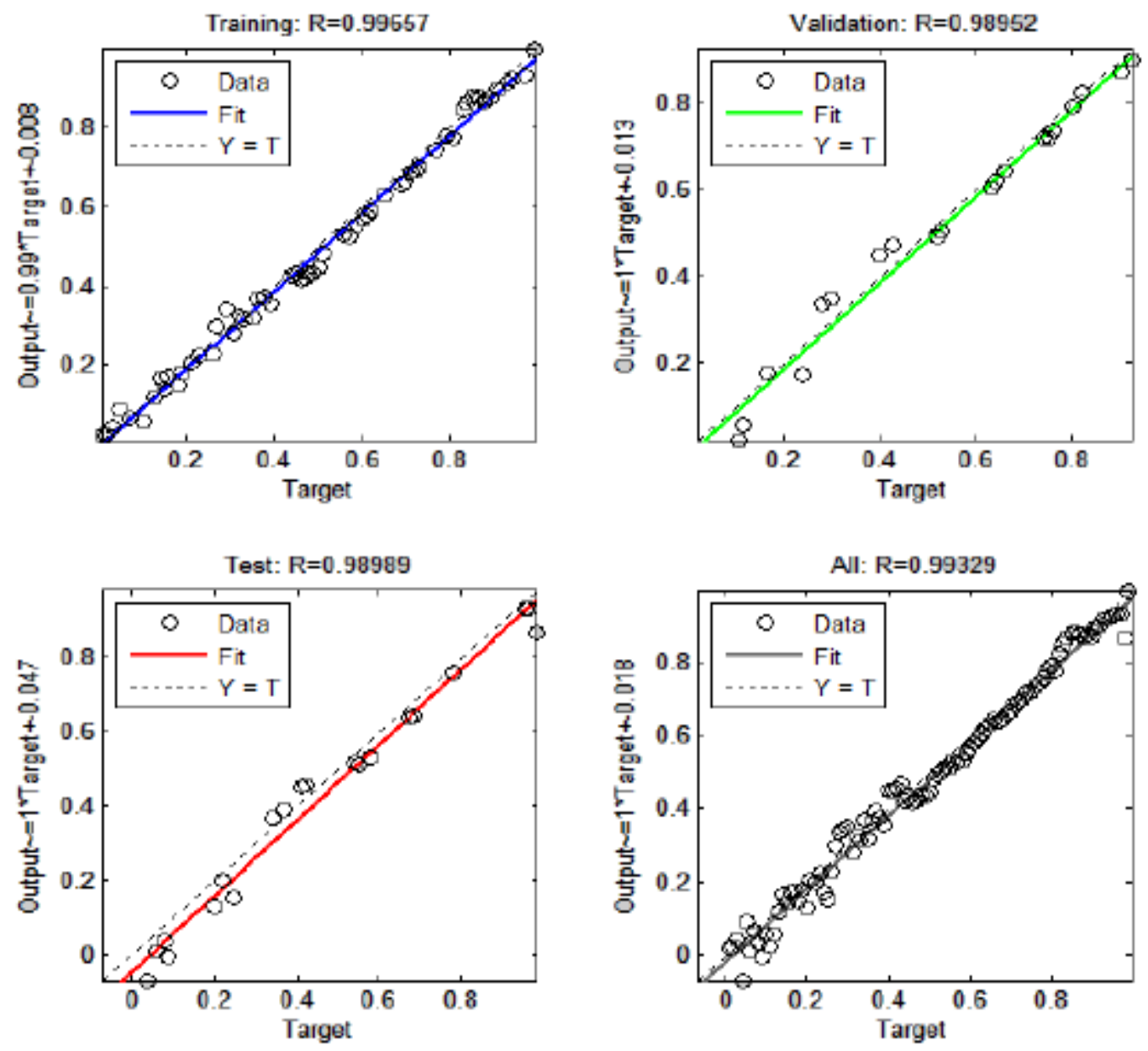

Figure 12: Regression plots of the various stages of learning of ANN (6-21-11-1).

\section{CONCLUSION}

Suitability of Artificial Neural Networks for fault detection and diagnosis of power systems is described in this research work. Various network topologies (number of hidden nodes and layers) have been tested and compared. The results show that accurate recall and generalization behaviors are obtained during the diagnosis of double faults. Performance during recall improves at first with an increase in the number of hidden nodes (units) and with the amount of training, and finally attains convergence. In general, performance during generalization improves with the duration of the training period. The neural network diagnostic system is also able to diagnose 
correctly even in the presence of faulty operation of the relays of the power system and under disturbances.

The neural network diagnostic system trained for double faults was found to be able to accurately diagnose abnormal behavior resulting from simultaneous multiple faults. Graceful degradation of the diagnostic system was observed in situations where faults where not accurately diagnosed or under damage to a few nodes.

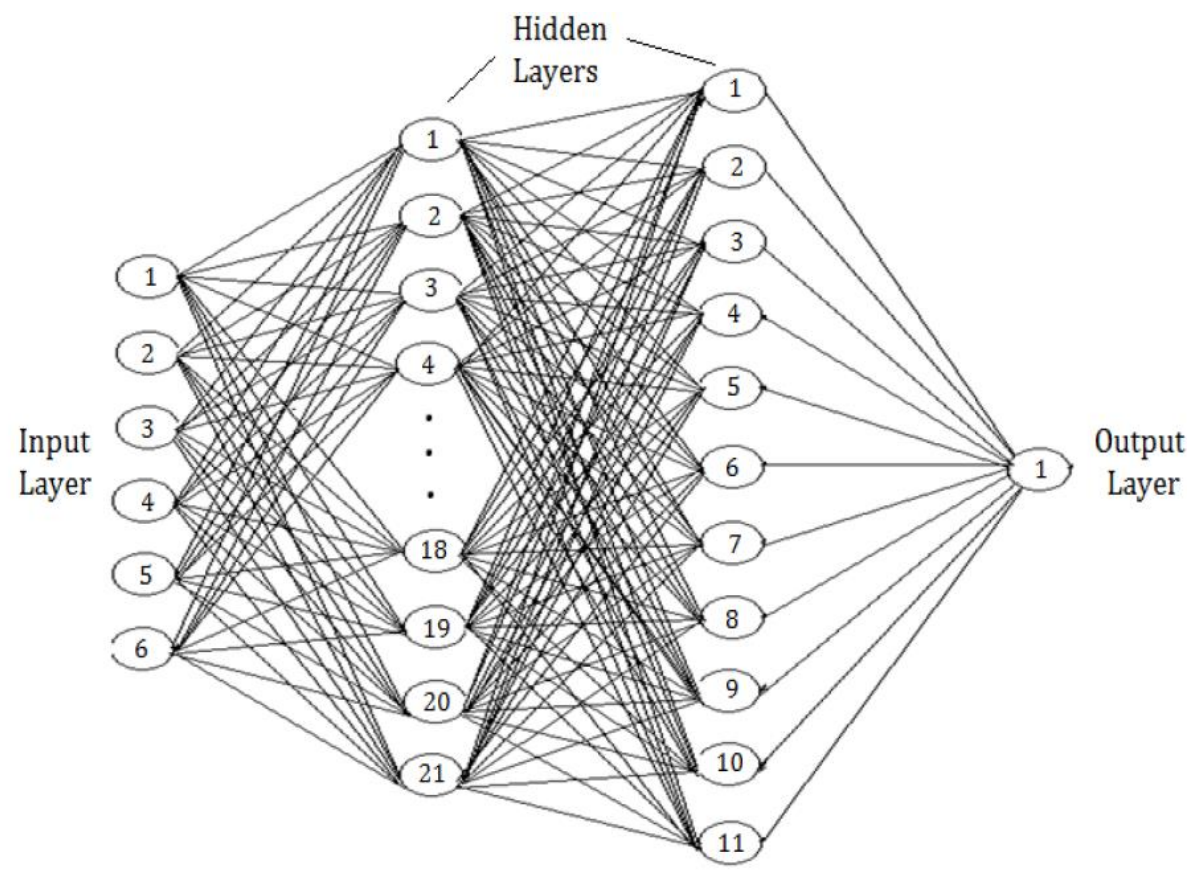

Figure 13: Structure of the chosen ANN $(6-21-11-1)$.

\section{REFERENCES}

[1] Zhang, Y., Zhang, Q., \& Song, W. (2013). "Transmission Line Fault Location for Double Phase to- Earth Fault on Non-Direct-Ground Neutral System." IEEE Transactions on Power Delivery, vol. 15(2), pp. 520-524.

[2] Cichoki, G. \& Unbehauen, H. (2013). "Numerical Distance Protection, Principles and Applications." Siemens AG, Publicis MCD Verlag, Erlangen. Pp 22-43.

[3] Kasztenny, C., Mohanta, D., \& Ferdimnand, V. (2011). "An Extended ANN-Based High Speed Accurate Distance Protection Algorithm.” Electric Power and Energy Systems, vol. 28(6), pp. $387-395$.

[4] Cook, D.A (2015). "A Fault Locator for Transmission Lines Using Travelling Waves and Wavelet Transform Theory." Proceedings of 8th International Conference on Developments in Power System Protection - DPSP, IEE, vol. CP500, pp. 212-215.

[5] Anderson, I. (2015). Network Protection \& Automation Guide. (2016). T\&D Energy Automation \& Information. Alstom, France.

[6] Eriksson, U., \& Rockefeller, A.S. (2015). "Autonomous Feature Discovery of Clearing Time Assessment." Symposium of Expert System Applications to Power Systems, Stockholm Helsinki, vol. 5, pp. 22-5.27. 
[7] Karl, R., \& David, E. (2015). “Adaptive-Neuro-Fuzzy Inference System Approach for Transmission Line Fault Classification and Location Incorporating Effects of Power Swings." Proceedings of IET Generation, Transmission and Distribution, vol. 6, pp. 235 - 244.

[8] Das, F., \& Novosel, G. (2013). "A Real-Time Hardware Fault Detector Using an Artificial Neural Network for Distance Protection.” IEEE Trans. on Power Delivery, vol. 16(1), pp. 75 - 82.

[9] Wanjing, X., \& Yuan, L. (2012). "Accurate Transmission Line Fault Location Considering Shunt Capacitances without Utilizing Line Parameters." Electric Power Components and Systems, vol. 43, pp. 12-19

[10] Girgis, SA., Yuan, L., \& Ning, G. (1992). "Electrical Power System Protection." Chapman \& Hall Publications, London. Vol. 3, pp. 54-81

[11] Saha, V.C., Yuan, L., \& Ivan, T. (2004). "Generalized Fault Location Methods for Overhead Electric Distribution Systems.” IEEE Transactions on Power Delivery, vol. 26(1), pp. 53-64.

[12] Takagi, W., Yuan, L., \& Ning, G. (1982). "Fault Location Algorithms Without Utilizing Line Parameters Based on Distributed Parameter Line Model.” IEEE Transactions on Power Delivery, vol. 24(2), pp. 579-584.

*Corresponding author.

E-mail address: ecnaxel@gmail.com 